\title{
A Synthetic Entry to Furo[2,3-b]pyridin-4(1H)-ones and Related Furoquinolinones via lodocyclization
}

\author{
I. Aillaud, E. Bossharth, D. Conreaux, \\ P. Desbordes, N. Monteiro, ${ }^{\star}$ and G. Balme*
}

General remarks: Unless otherwise noted, all reactions were carried out under a nitrogen atmosphere using standard syringe, cannula, and septa techniques. Commercially available reagents were used as purchased. THF and MeCN were distilled over calcium hydride. TLC was carried out on Merck silica 60/F-254 aluminiumbacked plates. Flash chromatography was performed using Merck silica gel $60(40-63 \mu \mathrm{m})$. Melting points were determined on a Büchi and are uncorrected. NMR spectra were recorded either on a Brucker ALS 300 or a Brucker AMS 200 spectrometers in the solvent indicated. Chemical shifts $\delta$ are reported in ppm relative to the internal reference. High resolution mass spectra were obtained on a Thermofinnigan LCQ-advantage or on a Thermofinnigan MAT95XL.

Preparation of the acetylenic precursors (1)

3-Alkynylpyridin-2(1H)-ones 1a-g and 3-alkynylquinolin-2(1H)-one $\mathbf{1 h}$ were prepared by reaction of the corresponding 3-iodo compounds with terminal acetylenes (Sonogashira coupling). Procedures for the preparation of the requisite 4-alkoxy-3-iodo-1-methylpyridin-2(1H)-ones [1, 2] and 3-iodo-4-methoxy-1methylquinolin-2(1H)-one [3] have been already reported.

Sonogashira coupling, general procedure: The iodo derivative $(0.6 \mathrm{mmol})$, the terminal alkyne $(0.8 \mathrm{mmol})$, $\mathrm{Pd}\left(\mathrm{PPh}_{3}\right)_{2} \mathrm{Cl}_{2}(21 \mathrm{mg}, 0.03 \mathrm{mmol})$ and $\mathrm{CuI}(5 \mathrm{mg}, 0.03 \mathrm{mmol})$ were dissolved in a mixture of $\mathrm{MeCN}(4 \mathrm{~mL})$ and TEA $(0.5 \mathrm{~mL})$. The reaction mixture was left to stir at $60^{\circ} \mathrm{C}$ for $24 \mathrm{~h}$ and then concentrated in vacuo. The residue was purified by column chromatography (silica gel, ethyl acetate) to afford the corresponding 3alkynyl-2-pyridone.

Compounds 1b, 1d, 1e, and $\mathbf{1 f}$ have been already described [1]

4-Methoxy-1-methyl-3-phenylethynylpyridin-2(1H)-one (1a): 81\%, solid, Mol. Wt.: 239.27 g/mol. Mp: 143$144{ }^{\circ} \mathrm{C} .{ }^{1} \mathrm{H} \mathrm{NMR}\left(300 \mathrm{MHz}, \mathrm{CDCl}_{3}\right): \delta 3.54(\mathrm{~s}, 3 \mathrm{H}), 3.94(\mathrm{~s}, 3 \mathrm{H}), 6.06(\mathrm{~d}, J=7.7 \mathrm{~Hz}, 1 \mathrm{H}), 7.28-7.32(\mathrm{~m}, 4 \mathrm{H})$, $7.56(\mathrm{dd}, J=2.1$ and $7.7 \mathrm{~Hz}, 2 \mathrm{H}) .{ }^{13} \mathrm{C} \mathrm{NMR}\left(75 \mathrm{MHz}, \mathrm{CDCl}_{3}\right): \delta .37 .35 ; 56.40 ; 82$; 94.09; 97.78; 98.11; 
123.68; 127.77; 128.00; 131.40; 138.94; 162.84; 168.58. HRMS (CI): $\mathrm{MH}^{+}, 240.1019$; Calc. for $\mathrm{C}_{15} \mathrm{H}_{14} \mathrm{NO}_{2}$ : 240.1018 .

3-(p-Hexyloxy)phenylethynyl-4-methoxy-1-methyl-2-pyridin-2(1H)-one (1c): (3 equiv of terminal alkyne were used). 82\%, oil, Mol. Wt.: $339.44 \mathrm{~g} / \mathrm{mol} .{ }^{1} \mathrm{H}$ NMR (300 MHz, $\left.\mathrm{CDCl}_{3}\right): \delta 0.85$ (t, $\left.J=6.4 \mathrm{~Hz}\right), 1.30-1.37$ $(\mathrm{m}, 4 \mathrm{H}), 1.39(\mathrm{t}, J=5.5 \mathrm{~Hz}, 2 \mathrm{H}), 1.71$ (quint, $J=6.8 \mathrm{~Hz}, 2 \mathrm{H}), 3.47(\mathrm{~s}, 3 \mathrm{H}), 3.86(\mathrm{~s}, 3 \mathrm{H}), 3.89$ (t, $J=6.5 \mathrm{~Hz}$, 2H), $6.00(\mathrm{~d}, J=7.7 \mathrm{~Hz}, 1 \mathrm{H}), 6.77(\mathrm{~d}, J=8.85 \mathrm{~Hz}, 2 \mathrm{H}) ; 7.29(\mathrm{~d}, J=7.7 \mathrm{~Hz}, 1 \mathrm{H}), 7.42(\mathrm{~d}, J=8.8 \mathrm{~Hz}, 2 \mathrm{H}) .{ }^{13} \mathrm{C}$ NMR (75 MHz, $\left.\mathrm{CDCl}_{3}\right): \delta .14 .00 ; 22.55 ; 25.66 ; 29.14 ; 31.54 ; 37.51 ; 56.50 ; 67.99 ; 80.47 ; 94.30 ; 98.26 ; 98.88$; 114.26; 115.75; 133.03; 138.39; 158.96; 163.05; 168.26. HRMS (EI): $\mathrm{M}^{+}$, 339.1837; Calc. for $\mathrm{C}_{21} \mathrm{H}_{25} \mathrm{NO}_{3}$ : 339.1834.

4-Methoxy-1-methyl-3-trimethylsilylethynyl-2-pyridin-2(1H)-one (1g): (3 equiv of terminal alkyne were used). 52\%, solid, Mol. Wt.: $239.27 \mathrm{~g} / \mathrm{mol}$. Mp: 123-124 ${ }^{\circ} \mathrm{C} .{ }^{1} \mathrm{H}$ NMR (300 MHz, $\left.\mathrm{CDCl}_{3}\right): \delta 0.21$ (s, 9H), 3.47 $(\mathrm{s}, 3 \mathrm{H}), 3.88(\mathrm{~s}, 3 \mathrm{H}), 6.00(\mathrm{~d}, J=7.7 \mathrm{~Hz}, 1 \mathrm{H}), 7.30(\mathrm{~d}, J=7.7 \mathrm{~Hz}, 1 \mathrm{H}) .{ }^{13} \mathrm{C} \mathrm{NMR}\left(75 \mathrm{MHz}, \mathrm{CDCl}_{3}\right): \delta .0 .63$; 38.02; 57.06; 94.63; 97.44; 98.; 104.17; 139.41; 163.86; 169.87. HRMS (EI): $\mathrm{M}^{+}, 235.1031$; Calc. for $\mathrm{C}_{12} \mathrm{H}_{17} \mathrm{NO}_{2} \mathrm{Si}: 235.1029$.

4-Methoxy-1-methyl-3-phenylethynylquinolin-2(1H)-one (1h): (3 equiv of terminal alkyne were used). 75\%, gum, Mol. Wt.: $289.34 \mathrm{~g} / \mathrm{mol} .{ }^{1} \mathrm{H}$ NMR $\left(300 \mathrm{MHz} \mathrm{CDCl}_{3}\right): \delta 3.72$ (s, 3H), 4.50 (s, 3H), 7.24 (t, $J=7.5 \mathrm{~Hz}$, 1H), 7.30-7.36 (m, 4H), 7.55-7.60 (m, 3H), $7.99(\mathrm{~d}, J=8.1 \mathrm{~Hz}, 1 \mathrm{H}) .{ }^{13} \mathrm{C} \mathrm{NMR}\left(75 \mathrm{MHz}, \mathrm{CDCl}_{3}\right): \delta .30 .32$; $61.22 ; 83.55 ; 99.28 ; 100.59 ; 114.38 ; 117.71 ; 122.48 ; 123.92 ; 124.43 ; 128.72 ; 128.78 ; 131.70 ; 132.10 ; 139.20$; 163.08; 164.74. HRMS (CI): $\mathrm{MH}^{+}, 290.1179$; Calc. for $\mathrm{C}_{19} \mathrm{H}_{16} \mathrm{NO}_{2}: 290.1181$.

Synthesis of the triiodide methosalts (2)

Iodocyclization, general procedure: The acetylene derivative $(0.2 \mathrm{mmol})$ was treated with iodine $(0.4 \mathrm{mmol})$ in $2 \mathrm{~mL} \mathrm{CH}_{2} \mathrm{Cl}_{2}$ and the reaction mixture was stirred at room temperature for $5 \mathrm{~h}$. The precipitated salt 2 was isolated by suction filtration, washed with dichloromethane $(10 \mathrm{~mL})$, and dried under vacuum. The brown filtrate was collected, washed with aqueous $\mathrm{Na}_{2} \mathrm{~S}_{2} \mathrm{O}_{3}$ and water, and dried $\left(\mathrm{MgSO}_{4}\right)$ to give a second crop of 2 after evaporation of the solvent.

3-Iodo-4-methoxy-7-methyl-2-phenylfuro[2,3-b]pyridinium triiodide (2a): solid, Mol. Wt.: 746.89 g/mol. Mp: $161-162{ }^{\circ} \mathrm{C} .{ }^{1} \mathrm{H}$ NMR (300 MHz, DMSO-d 6 ): $\delta 4.27$ (s, 3H), 4.28 (s, 3H), 7.59-7.65 (m, 4H), 8.16 (dd, $J=$ 1.7 and $7.5 \mathrm{~Hz}, 2 \mathrm{H}), 8.78$ (d, $J=7.3 \mathrm{~Hz}, 1 \mathrm{H}) .{ }^{13} \mathrm{C}$ NMR (75 MHz, DMSO-d 6 ): $\delta .39 .50 ; 57.89 ; 58.93 ; 105.36$; $114.91 ; 127.39 ; 127.73 ; 129.15 ; 130.90 ; 141.86 ; 152.55 ; 154.29 ; 166.03$. MS (ESI): $\mathrm{M}^{+}, 366$. 
3-Iodo-4-methoxy-2-(p-methoxycarbonyl)phenyl-7-methylfuro[2,3-b]pyridinium triiodide (2b): solid, Mol. Wt.: 804.93 g/mol. Mp: 146-147 ${ }^{\circ} \mathrm{C} .{ }^{1} \mathrm{H}$ NMR (300 MHz, DMSO-d 6 ): $\delta 3.91$ (s, 3H), 4.28 (s, 3H), 4.29 (s, $3 \mathrm{H}), 7.62(\mathrm{~d}, \mathrm{~J}=7.3 \mathrm{~Hz}, 1 \mathrm{H}), 8.19(\mathrm{~d}, J=8.3 \mathrm{~Hz}, 2 \mathrm{H}), 8.34(\mathrm{~d}, J=8.5 \mathrm{~Hz}, 2 \mathrm{H}), 8.81(\mathrm{~d}, J=7.3 \mathrm{~Hz}, 1 \mathrm{H}) .{ }^{13} \mathrm{C}$

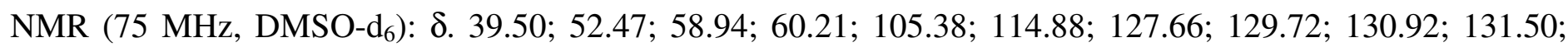
151.11; 154.30; 165.41; 166.27. MS (ESI): $\mathrm{M}^{+}, 424$.

2-(p-Hexyloxy)phenyl-3-iodo-4-methoxy-7-methylfuro[2,3-b]pyridinium triiodide (2c): solid, Mol. Wt.: 847.06 g/mol. Mp: 111-112 ${ }^{\circ} \mathrm{C} .{ }^{1} \mathrm{H}$ NMR (300 MHz, DMSO-d 6 ): $\delta 0.89$ (m, 3H), 1.33 (m, 4H), 1.43 (m, 2H), $1.75(\mathrm{~m}, 2 \mathrm{H}), 4.08(\mathrm{~m}, 2 \mathrm{H}), 4.26(\mathrm{~s}, 3 \mathrm{H}), 4.27(\mathrm{~s}, 3 \mathrm{H}), 7.19(\mathrm{~d}, \mathrm{~J}=8.5 \mathrm{~Hz}, 2 \mathrm{H}), 7.57(\mathrm{~d}, J=7.3 \mathrm{~Hz}, 1 \mathrm{H}), 8.10$ $(\mathrm{d}, J=8.5 \mathrm{~Hz}, 2 \mathrm{H}), 8.73(\mathrm{~d}, J=7.3 \mathrm{~Hz}, 1 \mathrm{H}) .{ }^{13} \mathrm{C} \mathrm{NMR}\left(75 \mathrm{MHz}, \mathrm{DMSO}-\mathrm{d}_{6}\right): \delta .13 .92 ; 22.06 ; 25.13 ; 28.51$; $30.97 ; 39.50 ; 55.47 ; 58.76 ; 67.87 ; 105.24 ; 114.99 ; 119.38 ; 129.42 ; 141.21 ; 152.86 ; 154.12 ; 160.55 ; 165.57$. MS (ESI): $\mathrm{M}^{+}, 466$.

4-Benzyloxy-3-iodo-7-methyl-2-phenylfuro[2,3-b]pyridinium triiodide (2d): solid, Mol. Wt.: 822.98 g/mol. Mp: $150{ }^{\circ} \mathrm{C}$ (dec.). ${ }^{1} \mathrm{H}$ NMR (300 MHz, DMSO-d 6 ): $\delta 4.28$ (s, 3H), 5.70 (s, 2H), 7.39-7.51 (m, 3H), 7.55-7.69 $(\mathrm{m}, 5 \mathrm{H}), 7.72(\mathrm{~d}, J=7.3 \mathrm{~Hz}, 1 \mathrm{H}), 8.16(\mathrm{~d}, J=8.8 \mathrm{~Hz}, 2 \mathrm{H}), 8.79(\mathrm{~d}, J=7.3 \mathrm{~Hz}, 1 \mathrm{H}) .{ }^{13} \mathrm{C} \mathrm{NMR}(75 \mathrm{MHz}$, DMSO-d 6 ): $\delta .40 .63 ; 55.08 ; 73.52 ; 106.90 ; 115.94 ; 128.25 ; 128.61 ; 128.9 ; 129.57 ; 129.61 ; 129.99 ; 131.75$; 135.23; 142.62; 153.54; 155.34; 165.81. Anal. Calcd for $\mathrm{C}_{21} \mathrm{H}_{17} \mathrm{I}_{4} \mathrm{NO}_{2}$ : C, 30.61; H, 2.20; N, 1.70. Found: C, 30.78; H, 2.09; N, 1.62 .

4-Benzyloxy-3-iodo-2-(p-methoxycarbonyl)phenyl-7-methylfuro[2,3-b]pyridinium triiodide (2e): solid, Mol. Wt.: 881.02 g/mol. Mp: $150{ }^{\circ} \mathrm{C}$ (dec.). ${ }^{1} \mathrm{H}$ NMR (300 MHz, DMSO-d 6 ): $\delta 3.91$ (s, 3H), 4.29 (s, $\left.3 \mathrm{H}\right), 5.71$ $(\mathrm{s}, 2 \mathrm{H}), 7.35-7.55(\mathrm{~m}, 3 \mathrm{H}), 7.64(\mathrm{~d}, J=6.8 \mathrm{~Hz}, 2 \mathrm{H}), 7.76(\mathrm{~d}, J=7.3 \mathrm{~Hz}, 1 \mathrm{H}), 8.20(\mathrm{~d}, J=8.6 \mathrm{~Hz}, 2 \mathrm{H}), 8.33(\mathrm{~d}$, $J=8.6 \mathrm{~Hz}, 2 \mathrm{H}), 8.81(\mathrm{~d}, J=7.3 \mathrm{~Hz}, 1 \mathrm{H}) .{ }^{13} \mathrm{C}$ NMR $\left(75 \mathrm{MHz}, \mathrm{DMSO}-\mathrm{d}_{6}\right): \delta .40 .63 ; 53.39 ; 61.35 ; 73.62$; $106.99 ; 115.98 ; 128.68 ; 128.91 ; 129.63 ; 130.69 ; 131.94 ; 132.48 ; 135.19 ; 143.18 ; 152.25 ; 155.51 ; 166.14$; 166.36. Anal. Calcd for $\mathrm{C}_{23} \mathrm{H}_{19} \mathrm{I}_{4} \mathrm{NO}_{4}$ : C, 31.36; H, 2.17; N, 1.59. Found: C, 31.36; H, 2.22; N, 1.59 .

4-Benzyloxy-2-butyl-3-iodo-7-methylfuro[2,3-b]pyridinium triiodide (2f): solid, Mol. Wt.: 802.99 g/mol. Mp: $139{ }^{\circ} \mathrm{C} .{ }^{1} \mathrm{H}$ NMR $\left(300 \mathrm{MHz}, \mathrm{DMSO}_{6}\right): \delta 0.93(\mathrm{t}, J=7.3 \mathrm{~Hz}, 3 \mathrm{H}), 1.38(\mathrm{~m}, 2 \mathrm{H}), 1.69(\mathrm{~m}, 2 \mathrm{H}), 2.91(\mathrm{t}, J$ $=7.3 \mathrm{~Hz}, 2 \mathrm{H}), 4.18(\mathrm{~s}, 3 \mathrm{H}), 5.65(\mathrm{~s}, 2 \mathrm{H}), 7.40-7.51(\mathrm{~m}, 3 \mathrm{H}), 7.61(\mathrm{~d}, J=6.6 \mathrm{~Hz}, 2 \mathrm{H}), 7.66(\mathrm{~d}, J=7.3 \mathrm{~Hz}, 1 \mathrm{H})$, $8.71(\mathrm{~d}, J=7.3 \mathrm{~Hz}, 1 \mathrm{H}) .{ }^{13} \mathrm{C}$ NMR $\left(75 \mathrm{MHz}, \mathrm{DMSO}_{6}\right): \delta .14 .46 ; 22.26 ; 27.56 ; 29.96 ; 40.57 ; 60.33 ; 73.23$; $106.64 ; 114.74 ; 128.76 ; 129.51 ; 129.57 ; 135.34 ; 141.93 ; 155.27 ; 160.29 ; 164.90$. Anal. Calcd for $\mathrm{C}_{19} \mathrm{H}_{21} \mathrm{I}_{4} \mathrm{NO}_{2}$ : C, 28.42; H, 3.85; N, 1.74. Found: C, 28.34; H, 3.60; N, 1.81.

3-Iodo-4-methoxy-9-methyl-2-phenylfuro[2,3-b]quinolinium triiodide (2h): solid, Mol. Wt.: 796.95 g/mol. Mp: $144-145^{\circ} \mathrm{C} .{ }^{1} \mathrm{H}$ NMR (300 MHz, DMSO-d $)$ ) $\delta 4.44$ (s, 3H), 4.56 (s, 3H), $7.69(\mathrm{~m}, 3 \mathrm{H}), 8.02(\mathrm{~m}, 1 \mathrm{H}), 8.33$ 
$(\mathrm{m}, 3 \mathrm{H}), 8.56(\mathrm{~d}, J=9.0 \mathrm{~Hz}, 1 \mathrm{H}), 8.63(\mathrm{~d}, J=8.1 \mathrm{~Hz}, 1 \mathrm{H}) .{ }^{13} \mathrm{C}$ NMR $\left(75 \mathrm{MHz}, \mathrm{DMSO}-\mathrm{d}_{6}\right): \delta .39 .50 ; 57.89$; $58.93 ; 105.36 ; 114.91 ; 127.39 ; 127.73 ; 129.15 ; 130.90 ; 141.86 ; 152.55 ; 154.29 ; 166.03$. MS (ESI): $\mathrm{M}^{+}, 416$.

Synthesis of the iodofurans (3)

general procedure: A mixture of the acetylene derivative $(0.2 \mathrm{mmol})$ and iodine $(0.4 \mathrm{mmol})$ in $2 \mathrm{~mL} \mathrm{MeCN}$ was stirred at room temperature for $5 \mathrm{~h}$, at which time $\mathrm{NaI}(0.3 \mathrm{mmol})$ was added. The mixture was refluxed overnight and then concentrated in vacuo, diluted with $\mathrm{CH}_{2} \mathrm{Cl}_{2}(10 \mathrm{~mL})$ and washed with aqueous $\mathrm{Na}_{2} \mathrm{~S}_{2} \mathrm{O}_{3}(2 \mathrm{x}$ $10 \mathrm{~mL})$. The aqueous layer was extracted with $\mathrm{CH}_{2} \mathrm{Cl}_{2}(2 \times 10 \mathrm{~mL})$ and the combined organic layers were dried $\left(\mathrm{MgSO}_{4}\right)$ and concentrated in vacuo to give the iodofuran as a solid of satisfactory purity.

3-Iodo-7-methyl-2-phenylfuro[2,3-b]pyridin-4(1H)-one (3a): solid, Mol. Wt.: 351.15 g/mol. Mp: 264-266 ${ }^{\circ} \mathrm{C} .{ }^{1} \mathrm{H}$ NMR $(300 \mathrm{MHz}$, DMSO-d 6 ): $\delta 3.78$ (s, 3H), $6.02(\mathrm{~d}, J=7.5 \mathrm{~Hz}, 1 \mathrm{H}), 7.45-7.61$ (m, 4H), 8.04 (d, $J=7.3$ $\mathrm{Hz}, 2 \mathrm{H}) .{ }^{13} \mathrm{C}$ NMR $\left(75 \mathrm{MHz}\right.$, DMSO-d $\left.{ }_{6}\right): \delta .27 .68 ; 50.99 ; 102.81 ; 105.70 ; 117.85 ; 119.95 ; 120.15 ; 120.30$; 128.20; 137.67; 144.59; 165.08. HRMS (CI): $\mathrm{MH}^{+}, 351.9832$; Calc. for $\mathrm{C}_{14} \mathrm{H}_{11} \mathrm{INO}_{2}: 351.9834$. Anal. Calcd for $\mathrm{C}_{14} \mathrm{H}_{10} \mathrm{INO}_{2}: \mathrm{C}, 47.89 ; \mathrm{H}, 2.87 ; \mathrm{N}, 3.99$. Found: C, 47.60; H, 2.87; N, 3.94 .

3-Iodo-2-(p-methoxycarbonyl)phenyl-7-methylfuro[2,3-b]pyridine-4(1H)-one (3b): solid, Mol. Wt.: 409.18 g/mol. Mp: 240-242 ${ }^{\circ} \mathrm{C}$ (dec.). ${ }^{1} \mathrm{H}$ NMR (300 MHz, DMSO-d d $_{\text {) }} \delta 3.83$ (s, 3H), 3.89 (s, 3H), 6.09 (d, $J=7.7$ $\mathrm{Hz}, 1 \mathrm{H}), 7.66(\mathrm{~d}, J=7.7 \mathrm{~Hz}, 1 \mathrm{H}), 8.09$ (d, $J=8.5 \mathrm{~Hz}, 2 \mathrm{H}), 8.23(\mathrm{~d}, J=8.5 \mathrm{~Hz}, 2 \mathrm{H}) .{ }^{13} \mathrm{C}$ NMR $(75 \mathrm{MHz}$, DMSO-d 6 ): $\delta .36 .67 ; 52.32 ; 62.82 ; 114.17 ; 126.17 ; 129.17 ; 127.56 ; 133.20 ; 137.51 ; 145.18 ; 153.70 ; 165.71$; 173.56. HRMS (EI): $\mathrm{M}^{+}, 408.9811$; Calc. for $\mathrm{C}_{16} \mathrm{H}_{12} \mathrm{INO}_{4}: 408.9811$.

2-(p-Hexyloxy)phenyl-3-iodo-7-methylfuro[2,3-b]pyridine-4(1H)-one (3c): solid, Mol. Wt.: $451.31 \mathrm{~g} / \mathrm{mol}$. Mp: 208-209 ${ }^{\circ} \mathrm{C} .{ }^{1} \mathrm{H}$ NMR (300 MHz, DMSO-d 6 ): $\delta 0.88$ (m, 3H), 1.31 (m, 4H), $1.42(\mathrm{~m}, 2 \mathrm{H}), 1.73(\mathrm{~m}, 2 \mathrm{H})$, 3.79 (s, 3H), $4.03(\mathrm{t}, J=6.4 \mathrm{~Hz}, 2 \mathrm{H}), 6.04(\mathrm{~d}, J=7.5 \mathrm{~Hz}, 1 \mathrm{H}), 7.08(\mathrm{~d}, J=8.8 \mathrm{~Hz}, 2 \mathrm{H}), 7.60(\mathrm{~d}, J=7.5 \mathrm{~Hz}$, $1 \mathrm{H}), 7.94(\mathrm{~d}, J=8.8 \mathrm{~Hz}, 2 \mathrm{H}) .{ }^{13} \mathrm{C}$ NMR $\left(75 \mathrm{MHz}, \mathrm{DMSO}-\mathrm{d}_{6}\right): \delta 13.92 ; 22.78 ; 25.17 ; 28.57 ; 31.00 ; 36.54$; $58.04 ; 67.67 ; 111.54 ; 114.08 ; 114.65 ; 121.15 ; 128.25 ; 136.84 ; 146.95 ; 153.06 ; 159.18 ; 173.51$. HRMS (CI): $\mathrm{MH}^{+}, 352.0723$; Calc. for $\mathrm{C}_{20} \mathrm{H}_{23} \mathrm{INO}_{3}: 452.0723$.

3-Iodo-9-methyl-2-phenylfuro[2,3-b]quinolin-4(9H)-one (3d): solid, Mol. Wt.: $401.21 \mathrm{~g} / \mathrm{mol}$. $\mathrm{Mp}>270^{\circ} \mathrm{C}$. $\mathrm{V}_{\max }(\mathrm{KBr}) 1635 \mathrm{~cm}^{-1} .{ }^{1} \mathrm{H}$ NMR $\left(300 \mathrm{MHz}, \mathrm{DMSO}-\mathrm{d}_{6}\right): \delta 3.97$ (s, 3H), 7.39-7.47 (m, 2H), 7.55 (t, $J=7.3 \mathrm{~Hz}$, $2 \mathrm{H}), 7.81(\mathrm{~m}, 2 \mathrm{H}), 8.11(\mathrm{~d}, J=7.5 \mathrm{~Hz}, 2 \mathrm{H}), 8.29(\mathrm{~d}, J=7.9 \mathrm{~Hz}, 1 \mathrm{H}) .{ }^{13} \mathrm{C}$ NMR $(75 \mathrm{MHz}$, DMSO-d 6 ): $\delta 31.53$; $60.58 ; 106.08 ; 115.70 ; 122.69 ; 125.04 ; 125.91 ; 126.18 ; 128.75 ; 129.01 ; 132.32 ; 137.67 ; 145.53 ; 155.10$; 171.60. HRMS (EI): $\mathrm{M}^{+}, 400.9914$; Calc. for $\mathrm{C}_{18} \mathrm{H}_{12} \mathrm{INO}_{2}: 400.9913$. 


\section{Coupling reactions of iodofuran 3a}

3-(p-Methoxycarbonyl)phenylethynyl-7-methyl-2-phenylfuro[2,3-b]pyridin-4(1H)-one (5): Iodofuran 3a (160 mg, $0.45 \mathrm{mmol})$, 4-methoxycarbonylphenyl acetylene (220 mg, $1.37 \mathrm{mmol}), \mathrm{PdCl}_{2}\left(\mathrm{PPh}_{3}\right)_{2}(26 \mathrm{mg}, 0.036$ $\mathrm{mmol})$ and $\mathrm{CuI}(7 \mathrm{mg}, 0.036 \mathrm{mmol})$ were dissolved in a mixture of $\mathrm{MeCN}(1 \mathrm{~mL})$ and TEA $(3 \mathrm{~mL})$. The reaction mixture was left to stir at $60^{\circ} \mathrm{C}$ for $72 \mathrm{~h}$ and then concentrated in vacuo. The residue was purified by column chromatography (silica gel, acetone) to afford $90 \mathrm{mg}$ (51\%) of compound 5: solid, Mol. Wt.: 383.37 g/mol. Mp: 216-218 ${ }^{\circ} \mathrm{C} .{ }^{1} \mathrm{H}$ NMR (300 MHz, DMSO-d $)$ ): $\delta 3.92(\mathrm{~s}, 3 \mathrm{H}), 3.93(\mathrm{~s}, 3 \mathrm{H}), 6.44(\mathrm{~d}, J=7.0 \mathrm{~Hz}, 1 \mathrm{H})$, $7.29(\mathrm{~d}, J=7.7 \mathrm{~Hz}, 1 \mathrm{H}), 7.38(\mathrm{~m}, 3 \mathrm{H}), 7.64(\mathrm{~d}, J=8.0 \mathrm{~Hz}, 2 \mathrm{H}), 8.01(\mathrm{~d}, J=8.0 \mathrm{~Hz}, 2 \mathrm{H}), 8.06(\mathrm{~d}, J=7.2 \mathrm{~Hz}$, 2H). ${ }^{13} \mathrm{C}$ NMR (75 MHz, DMSO-d 6 ): $\delta .36 .95 ; 52.36 ; 84.10 ; 95.43 ; 98.63 ; 111.65 ; 113.38 ; 125.06 ; 126.84$; $128.14 ; 129.20 ; 129.55 ; 129.70 ; 131.48 ; 138.32 ; 151.4 ; 153.11 ; 165.61 ; 172.05$. HRMS (CI): $\mathrm{MH}^{+}, 384.1236$; Calc. for $\mathrm{C}_{24} \mathrm{H}_{18} \mathrm{NO}_{4}: 384.1236$.

3-(p-Methoxy)phenyl-7-methyl-2-phenylfuro[2,3-b]pyridin-4(1H)-one (6a): A mixture of iodofuran 3a (70 $\mathrm{mg}, 0.2 \mathrm{mmol})$, 4-methoxyphenylboronic acid (91 mg, $0.6 \mathrm{mmol}), \mathrm{Pd}\left(\mathrm{PPh}_{3}\right)_{4}(11 \mathrm{mg}, 0.01 \mathrm{mmol})$, and $\mathrm{K}_{2} \mathrm{CO}_{3}$ $(83 \mathrm{mg}, 0.6 \mathrm{mmol})$ in a mixture of toluene $(2 \mathrm{~mL})$ and $\mathrm{EtOH}(1 \mathrm{~mL})$. The reaction mixture was stirred at $80^{\circ} \mathrm{C}$ for $16 \mathrm{~h}$ and then concentrated in vacuo. The residue was purified by column chromatography (silica gel, acetone/dichloromethane: $1 / 1)$ to afford $6 \mathbf{a}(66 \%)$ as a solid, Mol. Wt.: $331.37 \mathrm{~g} / \mathrm{mol}$. Mp> $200{ }^{\circ} \mathrm{C}$. ${ }^{1} \mathrm{H}$ NMR $\left(300 \mathrm{MHz}, \mathrm{CDCl}_{3}\right): \delta 3.73(\mathrm{~s}, 3 \mathrm{H}), 3.76(\mathrm{~s}, 3 \mathrm{H}), 6.13(\mathrm{~d}, J=7.5 \mathrm{~Hz}, 1 \mathrm{H}), 6.83(\mathrm{~d}, J=8.7 \mathrm{~Hz}, 2 \mathrm{H}), 7.02(\mathrm{~d}, J=$ 7.5 Hz, 1H), 7.10-7.20 (m, 3H), 7.35-7.45 (m, 4H). ${ }^{13} \mathrm{C}$ NMR (75 MHz, $\left.\mathrm{CDCl}_{3}\right)$ : $\delta . ~ 37.06 ; 55.62 ; 114.08$; $116.36 ; 120.65 ; 123.51 ; 126.80 ; 128.40 ; 128.86 ; 130.25 ; 132.14 ; 135.35 ; 145.04 ; 153.92 ; 159.69 ; 162.73$; 176.02. HRMS (CI): $\mathrm{MH}^{+}, 332.1287$; Calc. for $\mathrm{C}_{21} \mathrm{H}_{18} \mathrm{NO}_{3}: 332.1288$.

3-(p-Fluoro)phenyl-7-methyl-2-phenylfuro[2,3-b]pyridin-4(1H)-one $(\mathbf{6 b})$ : same procedure as for 6a $(89 \%$ yield); physical data identical to those previously reported [1].

[1] Bossharth, E.; Desbordes, P., Monteiro, N.; Balme, G. Org. Lett. 2003, 5, 2441.

[2] Conreaux, D.; Bossharth, E.; Desbordes, P., Monteiro, N.; Balme, G. Tetrahedron Lett. 2005, 46, 7917.

[3] Barr, S.; Neville, C. F; Grundon, M. F.; Boyd, D. R.; Malone, J. F.; Evans, T. A. J. Chem. Soc, Perkin Trans I 1995, 445. Reisch, J.; Marlies, I. Pharmazie 1993, 48, 579. Okuzumi, T.; Sagi, K.; Yoshimura, T.; Tanaka, Y.; Nakanishi, E.; Ono, M.; Murata, M.; PCT Int. Appl. WO03053926 (2003). 